\title{
Nl061490h: Supplementary Information
}

\section{Estimation of threshold gain coefficient for lasing}

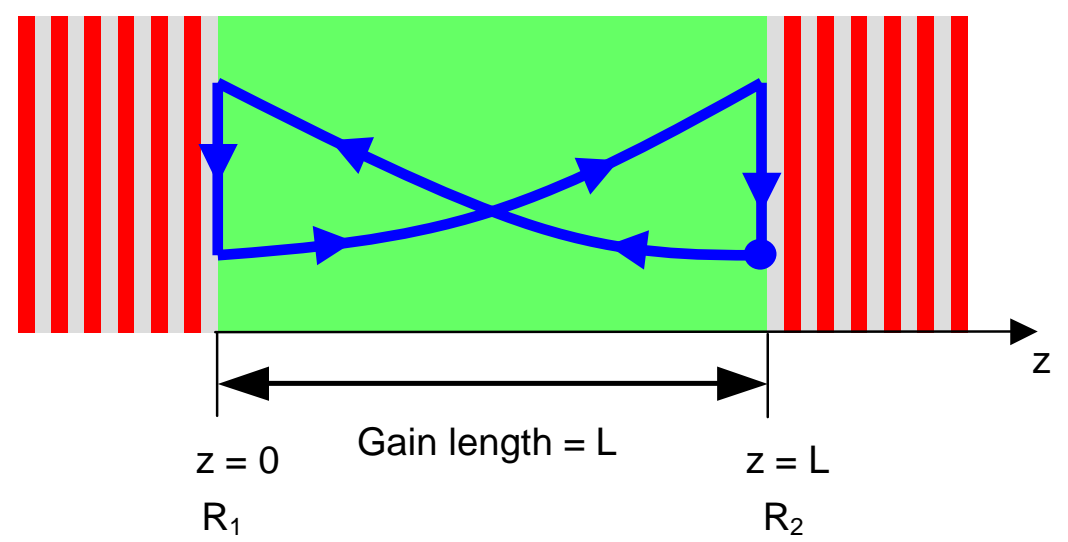

Figure S1: A model laser cavity composed of a gain medium (length L) and two dielectric mirrors having reflectivity values of $R_{1}(z=0)$ and $R_{2}(z=L)$. The blue arrow represents the power of the oscillating radiation.

Figure S1 shows a model laser cavity composed of a gain medium of the length $\mathrm{L}$ enclosed between two dielectric mirrors having reflectivity values of $R_{1}\left(\right.$ at $z=0$ ) and $R_{2}$ (at $\mathrm{z}=\mathrm{L}$ ) for a simple cavity analysis. As the spontaneous emission from the gain medium oscillates between two mirrors along the axis of the cavity, there exist both amplification (due to the gain) and attenuation (due to the absorption and the loss at mirrors) in the intensity of the oscillating radiation. Therefore, the power of the oscillating radiation, $\mathrm{P}(\mathrm{z})$, can be expressed as

$$
\mathrm{P}(\mathrm{z})=\mathrm{P}(0) \mathrm{e}^{\mathrm{Gz}} \mathrm{e}^{-\alpha z}
$$

where $P(0)$ is the power of the radiation at $z=0, G$ is the gain coefficient of the gain medium, $\alpha$ is the absorption coefficient of the gain medium. The steady-state oscillation condition then requires that the net gain must be the same as the net losses or the power of the radiation must be maintained constant on each round trip. Equation S2 shows a steadystate lasing condition for the cavity in Figure $S 1$, where $G_{t h}$ is the threshold gain coefficient of the gain medium. ${ }^{1}$ 


$$
\mathrm{e}^{\left(\mathrm{G}_{\mathrm{th}}-\alpha\right) \mathrm{L}} \mathrm{R}_{1} \mathrm{e}^{\left(\mathrm{G}_{\mathrm{th}}-\alpha\right) \mathrm{L}} \mathrm{R}_{2}=1
$$

The approximate threshold gain coefficient for lasing (neglecting absorption, $\alpha=0$ ) is then written in Equation S3.

$$
\mathrm{G}_{\mathrm{th}} \approx \frac{1}{2 \mathrm{~L}} \ln \left(\frac{1}{\mathrm{R}_{1} \mathrm{R}_{2}}\right)
$$

1. Siegman, A. E. Lasers (University Science Books, Palo Alto, 1986). 